\title{
Lasing in Optically Manipulated, Dye-doped Emulsion Microdroplets
}

\author{
M. Aas, A. Jonáśs*, and A. Kiraz* \\ Department of Physics, Koç University, Rumelifeneri Yolu, Sariyer, 34450 Istanbul, \\ Turkey \\ *Corresponding authors : ajonas@ku.edu.tr,akiraz@ku.edu.tr
}

\begin{abstract}
We introduce a portable, all-liquid microlaser based on optically pumped dye-doped emulsion microdroplets held in a single beam optical trap. We show high stability of the laser emission spectra during prolonged optical manipulation of the droplets within an immiscible host liquid. We investigate the effects of droplet size and dye concentration on the spectral position of lasing wavelength and show how these parameters can be used for the emission wavelength tuning. We also study shifting of the average lasing wavelength to the blue side of the spectrum due to dye photobleaching. The presented optically manipulated fluidic microlasers are disposable and can be easily combined with microfluidic chip technology. This makes them especially attractive for on-chip applications in chemical and biological analysis and sensing.
\end{abstract}

Keywords: microlaser; emulsion microdroplet; optical tweezers; whispering gallery mode; optofluidics.

\section{Introduction}

Liquid microdroplets can naturally serve as optical microcavities hosting high quality (high-Q) whispering gallery modes (WGMs), thanks to their selforganized spherical shape and smooth surface dictated by the surface tension of the liquid. Such high-Q cavities are a prerequisite for low-threshold lasing which is attractive in the development of integrated tunable organic light sources [1]. First demonstration of lasing from the liquid droplets dates back to 1984; in these pioneering experiments, lasing was observed in dye-doped 
free-falling ethanol droplets in air [2, 3]. Since then, numerous additional demonstrations of lasing in liquid droplets surrounded by air have followed. In particular, lasing has been observed in electrodynamically trapped dyedoped [4] and quantum dot-doped [5] droplets, ultrasonically trapped dyedoped droplets [6], or dye-doped droplets supported by a superhydrophobic surface [7]. In addition, Raman lasing in electrodynamically trapped [8] and surface-supported [9] droplets has been shown. Recently, in parallel with the advances in optofluidics, emulsion droplets surrounded by a suitable host liquid have also been studied as micro-lasers [10]. In such emulsion systems, fast switching of the emission color [11] and continuous tuning of the emission wavelength [12] have been presented.

In order to confine light inside a spherical liquid cavity, its index of refraction must be higher than the refractive index of the host medium. The requirement of the refractive index contrast also means that the droplets can be manipulated with a single-beam optical trap (optical tweezers) [13]. This opens up an exciting prospect of creating a microscopic laser source the position of which can be fully controlled by light. Optical tweezers have been used to manipulate emulsion droplets or droplets surrounded by air for microspectroscopy and chemical analysis applications [14, 15]. Despite these efforts, however, lasing in optically manipulated droplets surrounded by air or a liquid has not been demonstrated up to date; optically manipulated micro-lasers presented in the literature so far have always used solid spherical microcavities $[16,17]$

In this article we demonstrate lasing in dye-doped microdroplets of immersion oil emulsified in water that are manipulated by a single beam optical trap. We show that the laser emission spectrum can be tuned by changing the dye concentration and the droplet size. The presented optically manipulated droplet micro-laser is disposable and can be easily combined with microfluidic chip technology. Since water is selected as the host liquid, it is also compatible with biological applications.

\section{Description of Experiments}

Experimental setup for the observation of lasing from optically trapped emulsion microdroplets is shown in Figure 1. A continuous wave solid state infrared laser with $1064 \mathrm{~nm}$ wavelength and $300 \mathrm{~mW}$ maximum power (CrystaLaser) was used for microdroplet trapping. This laser was sent through a beam expander and reflected from two dichroic mirrors D1, D2 before being 
focused into the sample chamber by a water immersion microscope objective (Nikon, $\mathrm{NA}=1.2,60 \mathrm{x}$ ). In order to change the position of the optical trap in the objective focal plane, the first lens of the beam expander could be steered in $x$ and $y$ directions (perpendicular to the beam axis). The overall transmission of all optical elements in the trapping beam path was measured to be about $20 \%$, resulting in the average trapping power of $\sim 60 \mathrm{~mW}$ at the specimen. Fluorescently-stained trapped droplets were pumped with a $532 \mathrm{~nm}$ green beam obtained after second harmonic generation from a home-built passively Q-switched $\mathrm{Nd}-\mathrm{YVO}_{4}$ laser beam (20 ns pulse width and $33 \mathrm{kHz}$ repetition rate). The pump laser was focused by the same microscope objective as the trapping laser, after being transmitted through the dichroic mirror D1 and reflected from the dichroic mirror D2. Before the experiments, the droplet position was adjusted by moving the optical trap in the focal plane so that the focal spot of the pump laser lied near the droplet's rim for the maximal efficiency of the WGM excitation. Fluorescence from a trapped microdroplet was collected using the same microscope objective as used for focusing the trapping and pump beams, and dispersed by a monochromator (focal length $500 \mathrm{~mm}$; Acton Research) on the chip of a cooled CCD detector (Pixis 100; Princeton Instruments). In order to prevent fast photobleaching of dye molecules in the droplet, a shutter was added to the pump laser beam path; this shutter was only opened during the spectrum acquisition time and provided trigger signal for the spectroscopic detector to synchronize the data recording. The light in the imaging path of the microscope could be diverted to an ordinary CCD camera via a flippable mirror to observe the sample.

The emulsion system selected for our study consisted of microdroplets of dye-doped microscope immersion oil $\left(n=1.515, \rho=1.02 \mathrm{~g} / \mathrm{cm}^{3}\right.$, Merck) dispersed in deionized water $\left(n=1.334, \rho=1.00 \mathrm{~g} / \mathrm{cm}^{3}\right)$. This emulsion system of virtually immiscible liquids provides a high refractive index contrast together with a low density contrast between the droplet and the surrounding medium. This implies a good confinement of WGMs inside the trapped microdroplet, strong optical trapping force, and a small buoyancy that further facilitates the optical trapping of the droplets. In addition, hydrophobic nature of the immersion oil also prevents sticking of the microdroplets to the surfaces of regular cover glasses that were used to make the sample chamber. The dye DiI(3) (1,1'-Dioctadecyl-3,3,3',3'-tetramethylindo carbocyanine perchlorate, Sigma Aldrich) that has a hydrophilic chromophore and hydrophobic side chains was selected as the laser active medium. This dye has its excitation peak at $\sim 560 \mathrm{~nm}$ and emission peak at $\sim 575 \mathrm{~nm}$ (see also 
Fig. 5), and is well suited for the efficient excitation of the WGMs due to its surfactant nature that ensures its localization at the oil-water interface where WGMs also reside [18]. In our experiments DiI(3) was dissolved in chloroform with different concentrations and the dye solution was subsequently added to the immersion oil in 1 to 9 ratio. Final concentration of the dye in the droplets ranged from 0.1 to $1 \mathrm{mM}$. Addition of chloroform (refractive index 1.444) to the droplet liquid caused a small decrease of the droplet refractive index to the value of 1.506. Microdroplets were obtained by mixing the dyedoped immersion oil with water in 1 to 50 ratio, and shaking the obtained mixture by hand. Droplets produced this way had a large size distribution with diameters ranging from $<1$ up to $\sim 100 \mu \mathrm{m}$ and the emulsions were stable over $\sim 10$ hours. Prepared emulsions were subsequently loaded into a small capillary container made of two cover glasses attached to each other by double-sided tape. After loading, microdroplets were observed to float freely within the capillary container, thanks to their small buoyancy. The droplets did not stick to the walls of the sample chamber and could be readily trapped in all three dimensions.

The radius $a$ of the trapped droplets was calculated from the measured WGM spacing $\Delta \lambda$, mean lasing wavelength $\lambda$, and known refractive indices of the droplet $\left(n_{\text {int }}=1.506\right)$ and host $\left(n_{\text {ext }}=1.334\right)$ liquids, using the asymptotic formula of Chylek et al. [19]:

$$
a=\frac{\lambda^{2}}{2 \pi n_{\mathrm{ext}} \Delta \lambda} \frac{\arctan \left(\left[\left(n_{\mathrm{int}} / n_{\mathrm{ext}}\right)^{2}-1\right]^{1 / 2}\right)}{\left[\left(n_{\mathrm{int}} / n_{\mathrm{ext}}\right)^{2}-1\right]^{1 / 2}} .
$$

All droplet sizes presented in the following sections were determined from (1).

\section{Results and Discussions}

Figure 2 illustrates lasing from an optically trapped and manipulated droplet. In this figure, emission spectra and corresponding images of a $27 \mu \mathrm{m}$ diameter droplet with $1 \mathrm{mM}$ dye concentration are presented for different positions of the trapped droplet with respect to the sample chamber. All spectra are normalized by the same reference value - the overall maximum of emission intensity recorded at different droplet positions. Mean power of the pump beam used for recording the spectra was $1 \mathrm{~mW}$ (well above the lasing threshold - see below) and the data was acquired over the total 
trapping time of 10 min. A group of WGMs with large intensities centered at around $615 \mathrm{~nm}$ can be observed in the fluorescence spectra recorded at various droplet positions; these are the WGMs that exhibit lasing. Spectral region of droplet lasing is shifted from the emission maximum of the bulk dye solution at $\sim 575 \mathrm{~nm}$ towards the red end of the fluorescence spectrum to the location of the secondary emission peak of the dye (see Fig. 5). Here, the overall gain of the active medium given by an interplay between the spectral profile of the dye emission and the attenuation due to the dye self-absorption reaches maximum. In order to demonstrate the stability of optical confinement, sample chamber was translated past the trapped lasing droplet. Since the droplet was confined on the axis of a stationary trapping beam, its position in the field of view was fixed. Consequently, its displacement with respect to the sample chamber could be inferred from the image of a reference droplet resting on the sample chamber surface. In Figs. $2 \mathrm{a}$ and $2 \mathrm{~b}$, the trapped droplet is located approximately in contact with the bottom surface of the sample chamber while its transversal position along the surface changes. In Fig. 2c, the trapped droplet is lifted $\sim 50 \mu \mathrm{m}$ above its location in Fig. 2b, as indicated by defocusing of the reference droplet image. Comparison of the spectra corresponding to successive positions of the droplet in the sample chamber shows that the lasing modes are located at exactly the same wavelengths for all studied droplet positions. This implies that no change is observed in the size or the refractive index of the microdroplet over the whole duration of its manipulation.

In general, lasing can be observed only if the net gain of the active medium is larger than the cavity losses, which requires sufficiently high pumping. In order to determine the threshold pump power necessary for the droplet lasing, we analyzed a series of emission spectra recorded from a single optically trapped droplet with gradually increasing pump power. In Figure 3, the results of quantitative analysis of such spectral series acquired from a $31 \mu \mathrm{m}$ diameter droplet with $1 \mathrm{mM}$ dye concentration are presented.

Here, the intensities of both a lasing WGM at $620 \mathrm{~nm}$ and non-resonant fluorescence background in the immediate vicinity of the lasing WGM are shown as a function of the pump power. As illustrated by the graph, the intensities of the lasing WGM and the background grow with a different slope once the threshold pump power is exceeded which is indicative of the lasing onset. The value of the threshold pump power was estimated from the intersection of line fits to the background and lasing peak intensities, respectively. For the presented case, lasing threshold was around $70 \mu \mathrm{W}$, measured in the 
sample plane. It should be mentioned that due to the very short exposure time for each spectrum in the series ( $5 \mathrm{~ms}$ ), the influence of photobleaching on the measured emission intensities could be neglected; this is confirmed by the linear growth of background emission intensity over the whole range of the studied pump powers. Lasing threshold was investigated for different sizes of droplets. As an example, for droplets with 26 and $40 \mu \mathrm{m}$ diameters, threshold power of $\sim 140$ and $\sim 35 \mu \mathrm{W}$ was measured, respectively. The lower threshold power required for lasing in larger droplets can be attributed to their higher Q-factors [12].

Overall shape of the microdroplet lasing spectra depends on the absorption and emission profiles of the used dye, the concentration of the dye in the droplet, and the droplet size [12]. In order to elucidate the influence of these parameters on the droplet emission spectra, we carried out experiments with microdroplets of different sizes doped with different dye concentrations. The results of these experiments are summarized in Fig. 4 which reveals concentration- and size-dependent tuning of the average lasing wavelength. For the sake of clarity wavelength of the most intense lasing peak is given for each spectrum. In Fig. 4, pairs of spectra acquired from droplets of approximately equal sizes and dye concentrations differing by a factor of 10 are presented. Comparison of the spectra within each pair shows that the position of the most intense lasing peak is shifted to longer wavelengths for higher dye concentration. Such concentration-dependent shift of the lasing wavelength can be attributed to dye self-absorption. Figure 5 shows the normalized absorption and emission spectra of DiI(3) dye dissolved in chloroform/immersion oil mixtures at $1.25 \mu \mathrm{M}$ and $1 \mathrm{mM}$ concentrations, respectively. Absorption spectrum of the dye was acquired from a dilute dye solution placed in a spectroscopic cuvette. For recording emission spectra, a droplet of size similar to that used in the lasing experiments was pumped with the pulsed green beam the focus of which was placed at the droplet center so that the WGMs were not efficiently excited. Emission spectra were acquired both from a freshly generated droplet and from the same droplet after prolonged exposure to the green beam (400 ms). Prolonged irradiation with the green light caused photobleaching of the dye molecules and, thus, effectively lowered the dye concentration in the droplet (see below). Analysis of the absorption and emission spectra of $\mathrm{DiI}(3)$ reveals a spectral overlap at wavelengths immediately contiguous to the spectral region where the lasing modes are located. Consequently, part of the light emitted at shorter wavelengths is reabsorbed again by the dye molecules. Self-absorption phe- 
nomenon becomes more significant with increasing dye concentration and causes a shift of the effective emission maximum of the dye to the red end of the spectrum [20]. This tendency is clearly illustrated by comparing the emission spectra of a fresh droplet with high dye concentration (dashed line) and a partially photobleached droplet with low dye concentration (solid line). For a constant concentration of the fluorescent dye in the droplet, the position of the most intense lasing peak shifts to shorter wavelengths with decreasing droplet size. This trend can be qualitatively explained by considering the combined effect of the dye gain spectrum $g_{0}(\lambda)$, the efficiency of out-coupling of light from the droplet $\beta(a, \lambda)$, and the effective cross-section of the lasing WGMs $A(a, \lambda)$. The lasing power $P_{\text {out }}(a, \lambda)$ coupled out of the droplet scales as $[21]$

$$
P_{\text {out }}(a, \lambda) \sim \frac{A(a, \lambda)}{\lambda^{3}} g_{0}(\lambda) \beta(a, \lambda) .
$$

While $g_{0}$ is independent of the droplet size, both $\beta$ and $A$ change with the droplet radius $a$. As argued by Tang et al. in [12], for the studied experimental situation, the maximum of the product of $\beta(a, \lambda)$ and $A(a, \lambda) / \lambda^{3}$ shifts to the blue end of the spectrum for smaller droplets. Hence, optimal conditions for droplet lasing at a particular dye concentration that are given by the spectral position of the maximum of (2) occur at shorter wavelengths. When comparing the lasing spectra obtained with different dye concentrations, the sizes of the droplets used in the comparison were not identical. Thus, in principle, both concentration and size variations contributed to the observed differences in the spectral position of the lasing maximum. However, since the relative size differences within the studied droplet pairs were small (less than $6 \%$ ), the effect of the concentration change was dominant.

Upon prolonged exposure to pump light, dye molecules inside the droplet undergo photobleaching that decreases the effective concentration of absorbing chromophores in the droplet. This in turn leads to changes of the emission spectral profile of the dye. In Fig. 5, normalized emission spectra of droplets containing $1 \mathrm{mM} \operatorname{DiI}(3)$ solution excited with $532 \mathrm{~nm}$ pulsed laser beam with $\sim 300 \mu \mathrm{W}$ average power are presented. These spectra were acquired immediately after the start of the experiment, during the first $5 \mathrm{~ms}$ exposure with the green beam (dashed line), and after $400 \mathrm{~ms}$ exposure with the green beam when partial photobleaching had taken place (solid line). Lowering of dye concentration due to photobleaching causes a decrease of self-absorption and, thus, an overall blue shift in the observed emission spectrum. This 
observation is consistent with Fig. 4 where a decrease of the dye concentration causes shift of the lasing emission maximum to the blue end of the spectrum. As a result of the photobleaching process, the droplet emission profile evolves in time. Figure 6 illustrates quantitatively the dynamics of photobleaching. The spectra of Fig. 6 were recorded from a single droplet of diameter $\sim 34 \mu \mathrm{m}$ with the initial dye concentration $1 \mathrm{mM}$ and the average pump power of $1.8 \mathrm{~mW}$. As expected, the overall intensity of the spectra decreases with increasing frame number (bottom to top). Moreover, as the photobleaching progresses, spectral location of the most intense lasing peak moves to shorter wavelengths; in the presented spectral series, the maximal lasing intensity shifts from peak labeled C to B to A between the 1st, 9th and 19th frame, respectively. This trend is a direct consequence of the blue shift in the overall dye emission spectrum due to photobleaching, as shown in Fig. 5. However, while the location of the lasing maximum changes in time, the spectral positions of individual WGMs remain constant. This indicates that the droplet size and refractive index do not change during the experiment. Thus, prolonged confinement of the droplet in the optical trap does not influence its optical parameters; we observed stable WGM structures from droplets that were trapped for up to several hours. It should be mentioned that the bleaching rate at a fixed pump power depends strongly on the droplet size; it is possible to acquire more spectra from bigger droplets before they bleach. For example, with $1 \mathrm{mM}$ dye concentration at $1.8 \mathrm{~mW}$ average pump power and $5 \mathrm{~ms}$ acquisition time, the droplets with the diameter of $\sim 26 \mu \mathrm{m}, \sim 31 \mu \mathrm{m}$, and $\sim 53 \mu \mathrm{m}$ bleach after acquiring 50, 100, and 200 spectral frames, respectively.

\section{Conclusions}

In this article, we introduced and characterized an optically manipulated dye micro-laser based on liquid droplet immersed in an immiscible host fluid. We demonstrated the stability of laser emission during the droplet positioning within the host liquid and showed that the laser emission wavelength can be tuned by changing the droplet size and/or the concentration of fluorescent dye in the droplet. Emulsion droplet micro-lasers of precise sizes can be easily generated from various fluid combinations using microfluidic chips. This makes them especially attractive for on-chip applications in chemical and biological analysis and sensing. After the droplet bleaches, it can be easily replaced with a fresh droplet generated on demand which is a clear 
advantage over solid-state spherical microcavities. Moreover, in contrast to the solid-state cavities, shape of the liquid droplets can be easily deformed which provides further potential for reversible tuning of the laser emission spectra.

\section{Acknowledgment}

This work was partially supported by TÜBİTAK (Grant No. 111T059) and European Commission Marie Curie IEF (Contract No. PIEF-GA-2009252579 ; A.J.).

[1] K. Mølhave, A. Kristensen, N. A. Mortensen, Advanced Photonic Structures for Biological and Chemical Detection, Springer, pp. 471-486.

[2] H.-M. Tzeng, K. F. Wall, M. B. Long, R. K. Chang, Opt. Lett. 9 (1984) 499-501.

[3] S.-X. Qian, J. B. Snow, H. M. Tzeng, R. K. Chang, Science 231 (1986) 486.

[4] M. Tona, M. Kimura, J. Phys. Soc. Jpn. 69 (2000) 3533-3535.

[5] J. Schäfer, J. P. Mondia, R. Sharma, Z. H. Lu, A. S. Susha, A. L. Rogach, L. J. Wang, Nano Lett. 8 (2008) 1709-1712.

[6] H. Azzouz, L. Alkhafadiji, S. Balslev, J. Johansson, N. A. Mortensen, S. Nilsson, A. Kristensen, Opt. Express 14 (2006) 4374-4379.

[7] A. Kiraz, A. Sennaroglu, S. Doganay, M. A. Dundar, A. Kurt, H. Kalaycoglu, A. L. Demirel, Opt. Commun. 276 (2007) 145-148.

[8] R. Sharma, J. P. Mondia, J. Schäfer, Z. H. Lu, L. J. Wang, J. Appl. Phys. 105 (2009) 113104.

[9] A. Sennaroglu, A. Kiraz, M. A. Dündar, A. Kurt, A. L. Demirel, Opt. Lett. 32 (2007) 2197-2199.

[10] M. Tanyeri, R. Perron, I. M. Kennedy, Opt. Lett. 32 (2007) 2529-2531.

[11] S. K. Y. Tang, Z. Li, A. R. Abate, J. J. Agresti, D. A. Weitz, D. Psaltis, G. M. Whitesides, Lab on a Chip 9 (2009) 2767-2771. 
[12] S. K. Y. Tang, R. Derda, Q. Quan, M. Loncar, G. M. Whitesides, Opt. Express 19 (2011) 2204-2215.

[13] A. Ashkin, J. M. Dziedzic, J. E. Bjorkholm, S. Chu, Opt. Lett. 11 (1986) 288-290.

[14] N. Kitamura, F. Kitagawa, Journal of Photochemistry and Photobiology C: Photochemistry Reviews 4 (2003) 227-247.

[15] J. P. Reid, H. Meresman, L. Mitchem, R. Symes, Int. Rev. Phys. Chem. 26 (2007) 139-192.

[16] K. Sasaki, H. Fujiwara, H. Masuhara, J. Vac. Sci. Technol. B 15 (1997) 2786-2790.

[17] K. Sasaki, H. Fujiwara, H. Masuhara, Appl. Phys. Lett. 70 (1997) 26472649.

[18] S. Holler, N. L. Goddard, S. Arnold, J. Chem. Phys. 108 (1998) 65456547.

[19] P. Chylek, J. T. Kiehl, M. K. W. Ko, Physical Review A: Atomic, Molecular, and Optical Physics 18 (1978) 2229.

[20] D. V. Vezenov, B. T. Mayers, D. B. Wolfe, G. M. Whitesides, Appl. Phys. Lett. 86 (2005) 041104.

[21] H. B. Lin, J. D. Eversole, A. J. Campillo, J. Opt. Soc. Am. B 9 (1992) $43-50$. 


\section{Figure Captions}

\section{Figure 1}

Experimental setup for microdroplet lasing studies. The inset shows the detail of an optically trapped droplet with the pump laser beam positioned on the droplet rim. D1, D2 - dichroic mirrors, F - band-pass filter, FM flippable mirror, L1, L2 - lenses, M - mirror, PBS - polarizing beam splitter, WP - lambda-half wave plate.

\section{Figure 2}

Laser emission spectra from an optically manipulated emulsion droplet (diameter $27 \mu \mathrm{m}$; denoted by a dashed circle). Arrows indicate a stationary reference droplet resting on the bottom surface of the sample chamber. The trapped droplet is first translated in the plane of the figure between (a)-(b) and subsequently out of the figure plane between (b)-(c).

\section{Figure 3}

Dependence of the droplet emission intensity on the input pump power. Intensities of both a lasing mode at $\sim 620 \mathrm{~nm}$ and non-resonant fluorescence background in the immediate vicinity of the mode are shown. Inset identifies respective spectral locations for the measured intensity values. Droplet diameter for this measurement was $31 \mu \mathrm{m}$.

\section{Figure 4}

Dependence of the lasing wavelength on the droplet size and the dye concentration inside the droplet. Tags of individual spectra provide the droplet size, the dye concentration, and the wavelength of the most intense lasing peak. For both studied dye concentrations, droplets of approximately equal size were analyzed (see color coding of the spectra). Average pump power and exposure time for each frame in this measurement were $0.7 \mathrm{~mW}$ and 6 ms, respectively.

\section{Figure 5}

Normalized absorption (dotted line) and emission spectra of DiI(3) dye dissolved in immersion oil/chloroform mixture. The emission spectra are shown at two different levels of dye photobleaching (dashed line - initial dye emission, solid line - dye emission after $400 \mathrm{~ms}$ exposure with a $532 \mathrm{~nm}$ pulsed green laser with $\sim 300 \mu \mathrm{W}$ average power).

\section{Figure 6}

Effect of photobleaching on the droplet lasing spectra. 1st, 9th, and 19th frames of spectra acquired from a $\sim 34 \mu \mathrm{m}$ diameter droplet are shown. Average pump power was $1.8 \mathrm{~mW}$ and exposure time for each of the frames was $10 \mathrm{~ms}$. 
FIGURE 1, Aas et al.

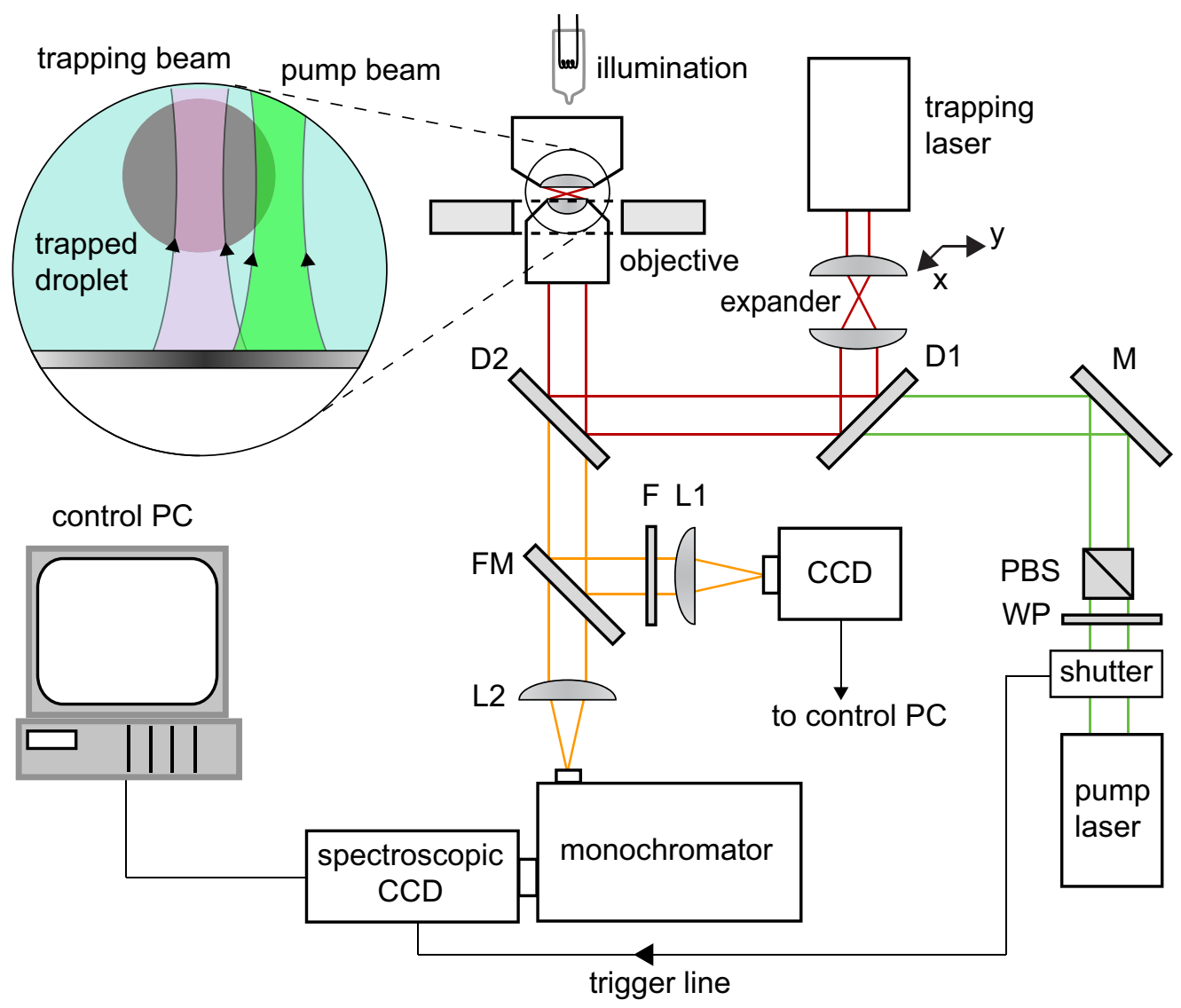

Figure 1: Experimental setup for microdroplet lasing studies. The inset shows the detail of an optically trapped droplet with the pump laser beam positioned on the droplet rim. D1, D2 - dichroic mirrors, F - band-pass filter, FM - flippable mirror, L1, L2 - lenses, M mirror, PBS - polarizing beam splitter, WP - lambda-half wave plate. 
FIGURE 2, Aas et al.

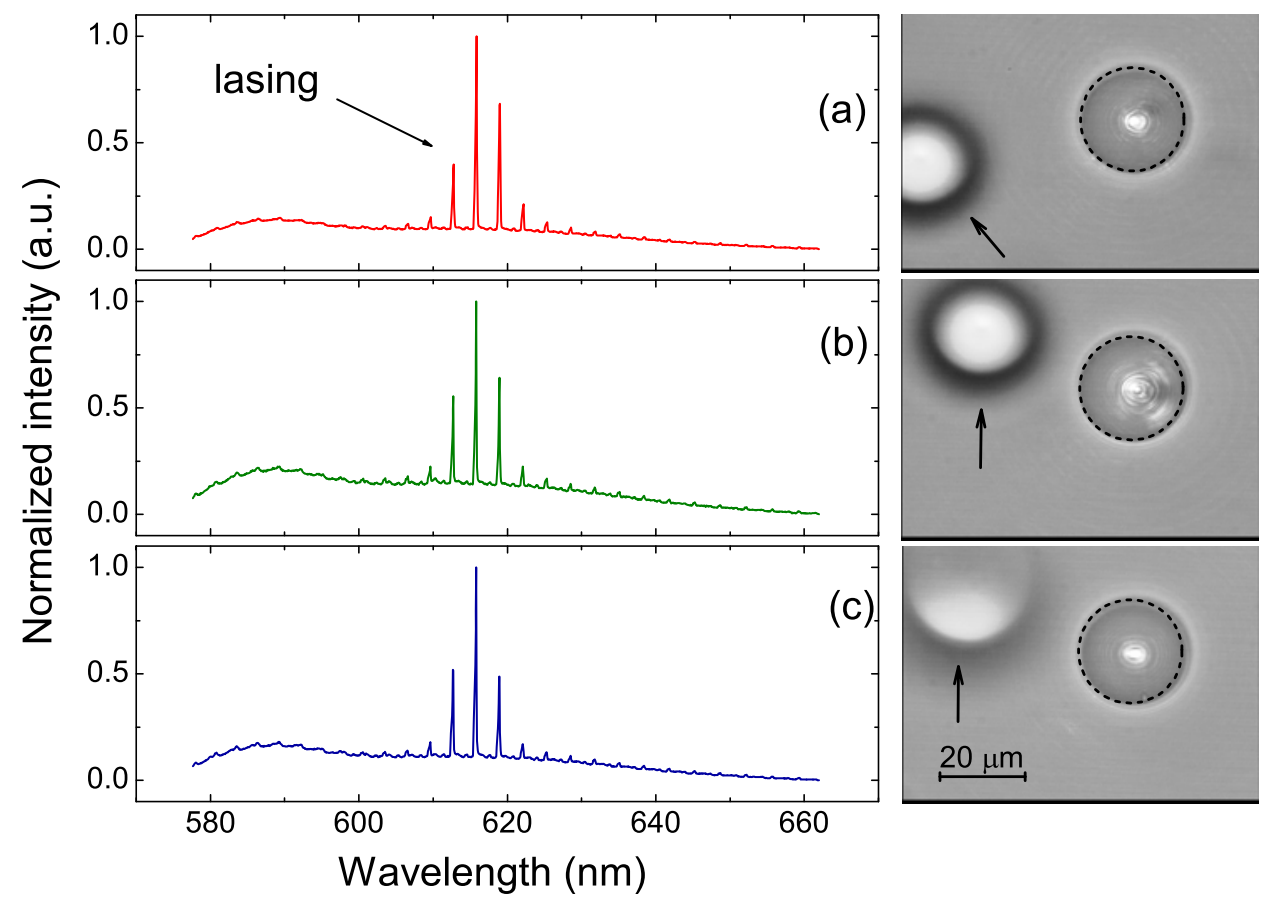

Figure 2: Laser emission spectra from an optically manipulated emulsion droplet (diameter $27 \mu \mathrm{m}$; denoted by a dashed circle). Arrows indicate a stationary reference droplet resting on the bottom surface of the sample chamber. The trapped droplet is first translated in the plane of the figure between (a)-(b) and subsequently out of the figure plane between (b)-(c). 
FIGURE 3, Aas et al.

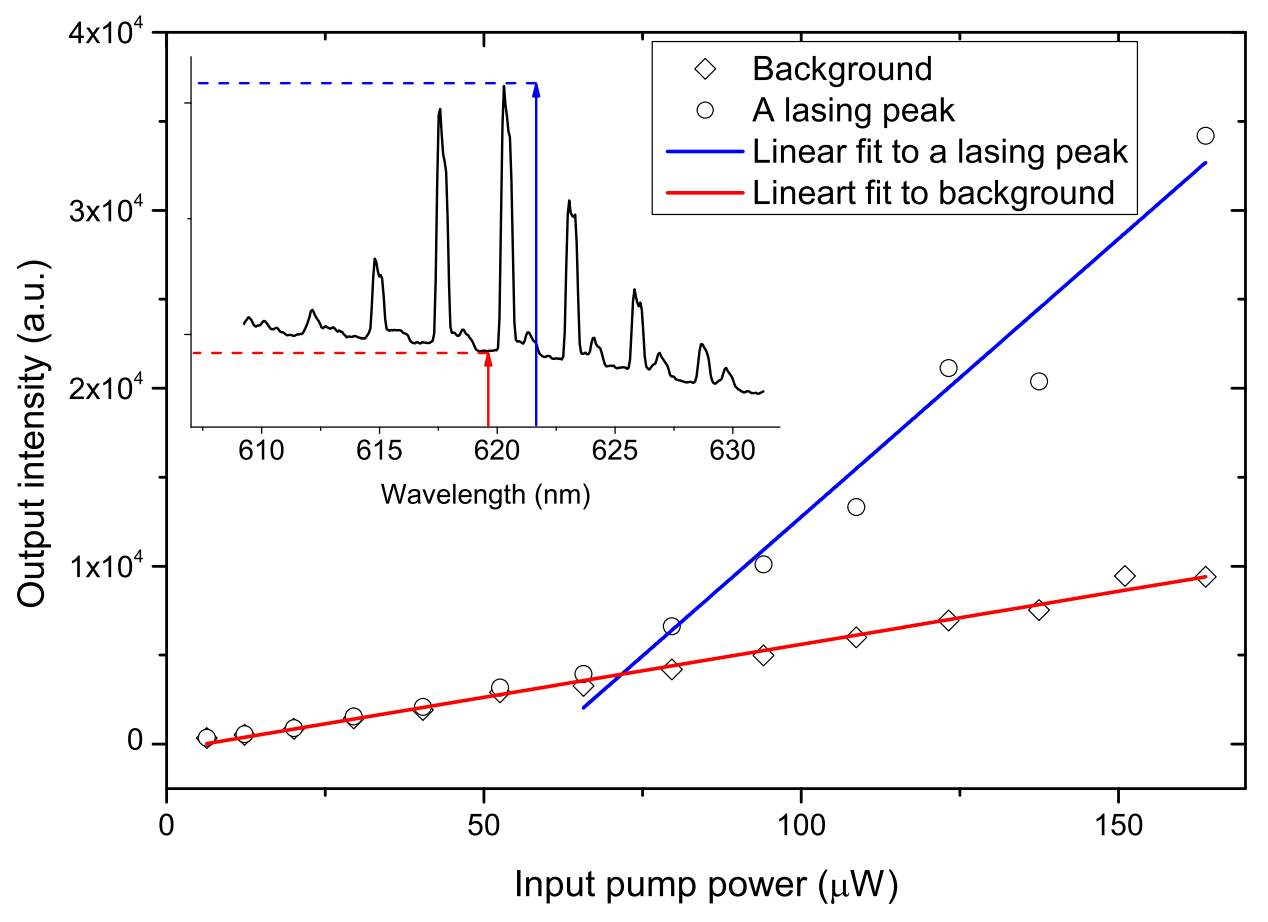

Figure 3: Dependence of the droplet emission intensity on the input pump power. Intensities of both a lasing mode at $\sim 620 \mathrm{~nm}$ and non-resonant fluorescence background in the immediate vicinity of the mode are shown. Inset identifies respective spectral locations for the measured intensity values. Droplet diameter for this measurement was $31 \mu \mathrm{m}$. 
FIGURE 4, Aas et al.

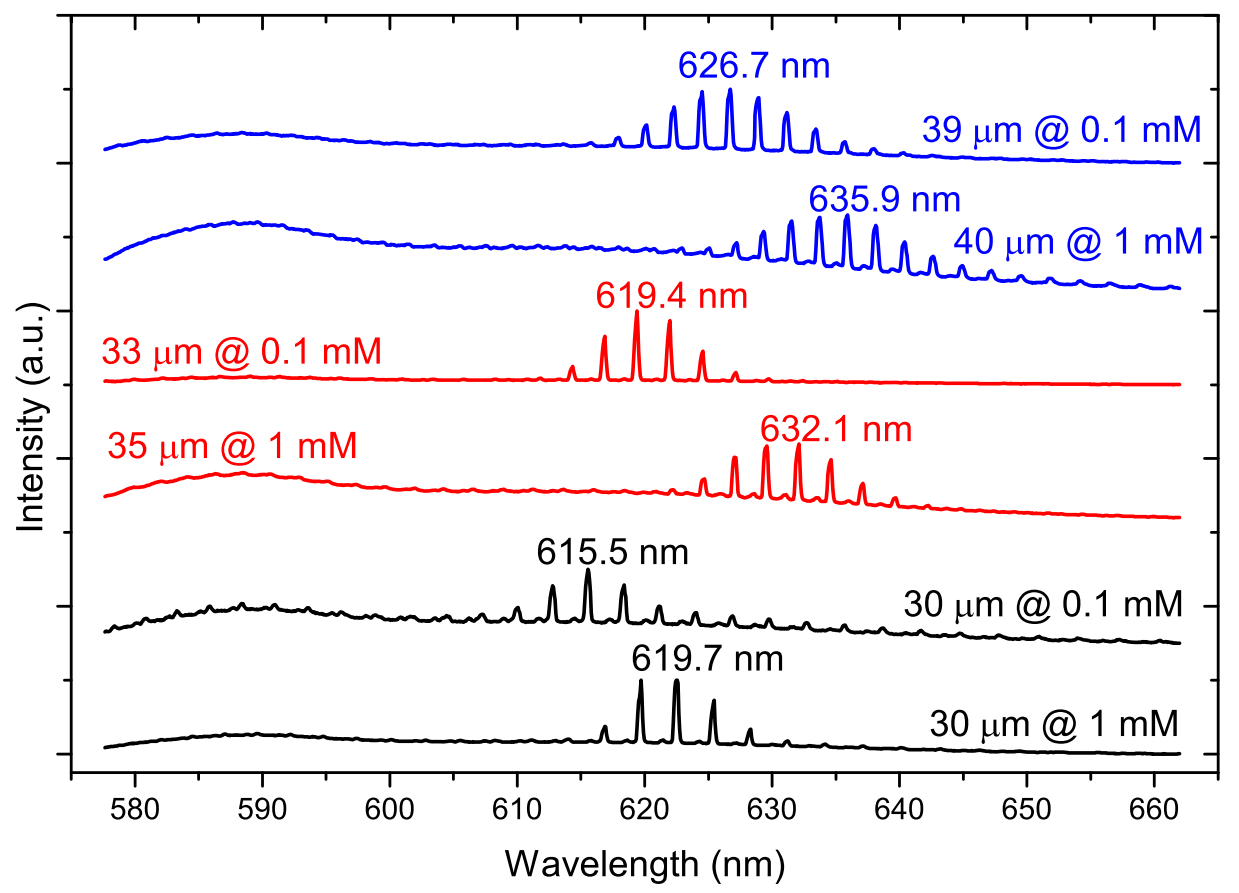

Figure 4: Dependence of the lasing wavelength on the droplet size and the dye concentration inside the droplet. Tags of individual spectra provide the droplet size, the dye concentration, and the wavelength of the most intense lasing peak. For both studied dye concentrations, droplets of approximately equal size were analyzed (see color coding of the spectra). Average pump power and exposure time for each frame in this measurement were $0.7 \mathrm{~mW}$ and $6 \mathrm{~ms}$, respectively. 
FIGURE 5, Aas et al.
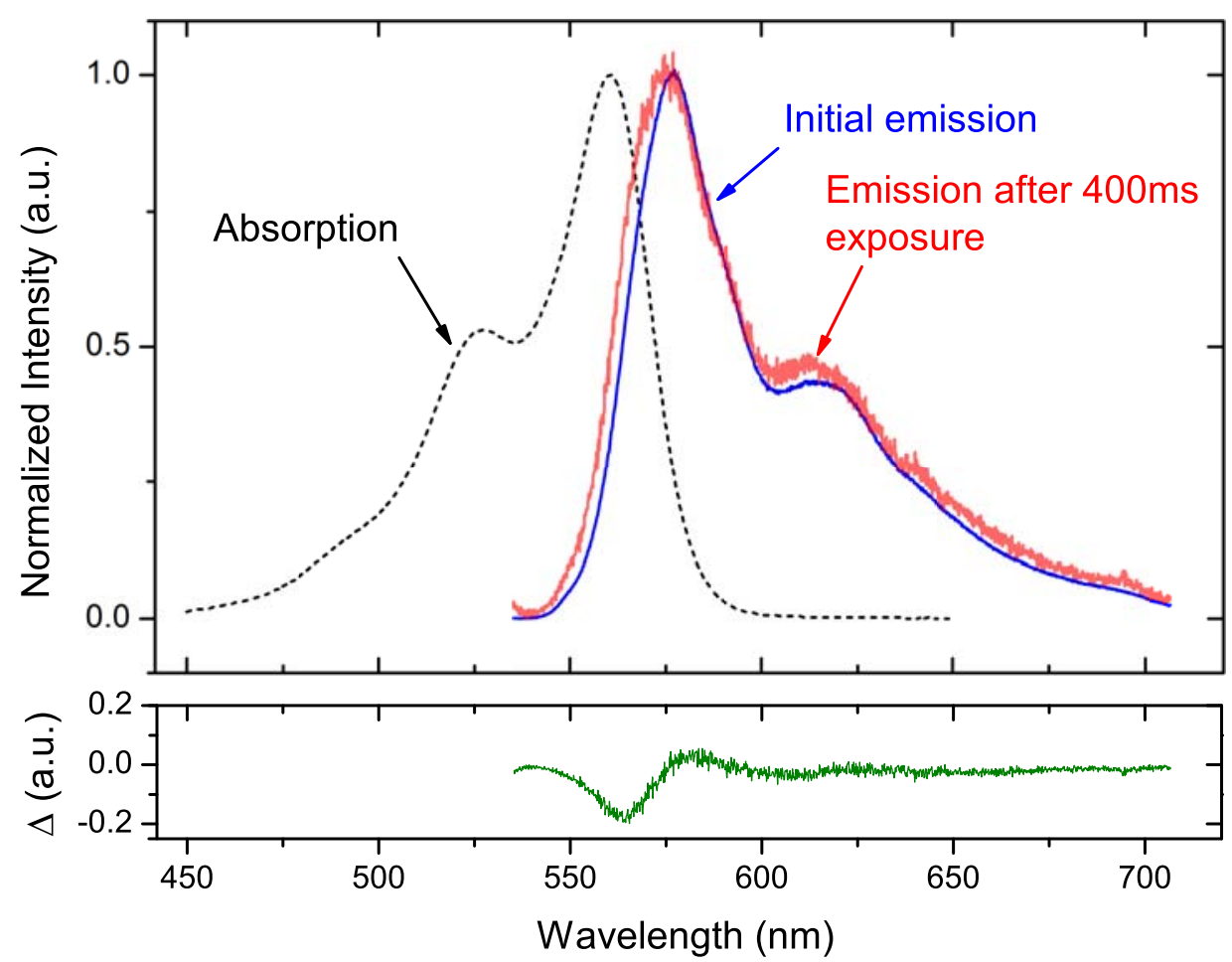

Figure 5: Normalized absorption (dotted line) and emission spectra of DiI(3) dye dissolved in immersion oil/chloroform mixture. The emission spectra are shown at two different levels of dye photobleaching (dashed line - initial dye emission, solid line - dye emission after $400 \mathrm{~ms}$ exposure with a $532 \mathrm{~nm}$ pulsed green laser with $\sim 300 \mu \mathrm{W}$ average power). 
FIGURE 6, Aas et al.

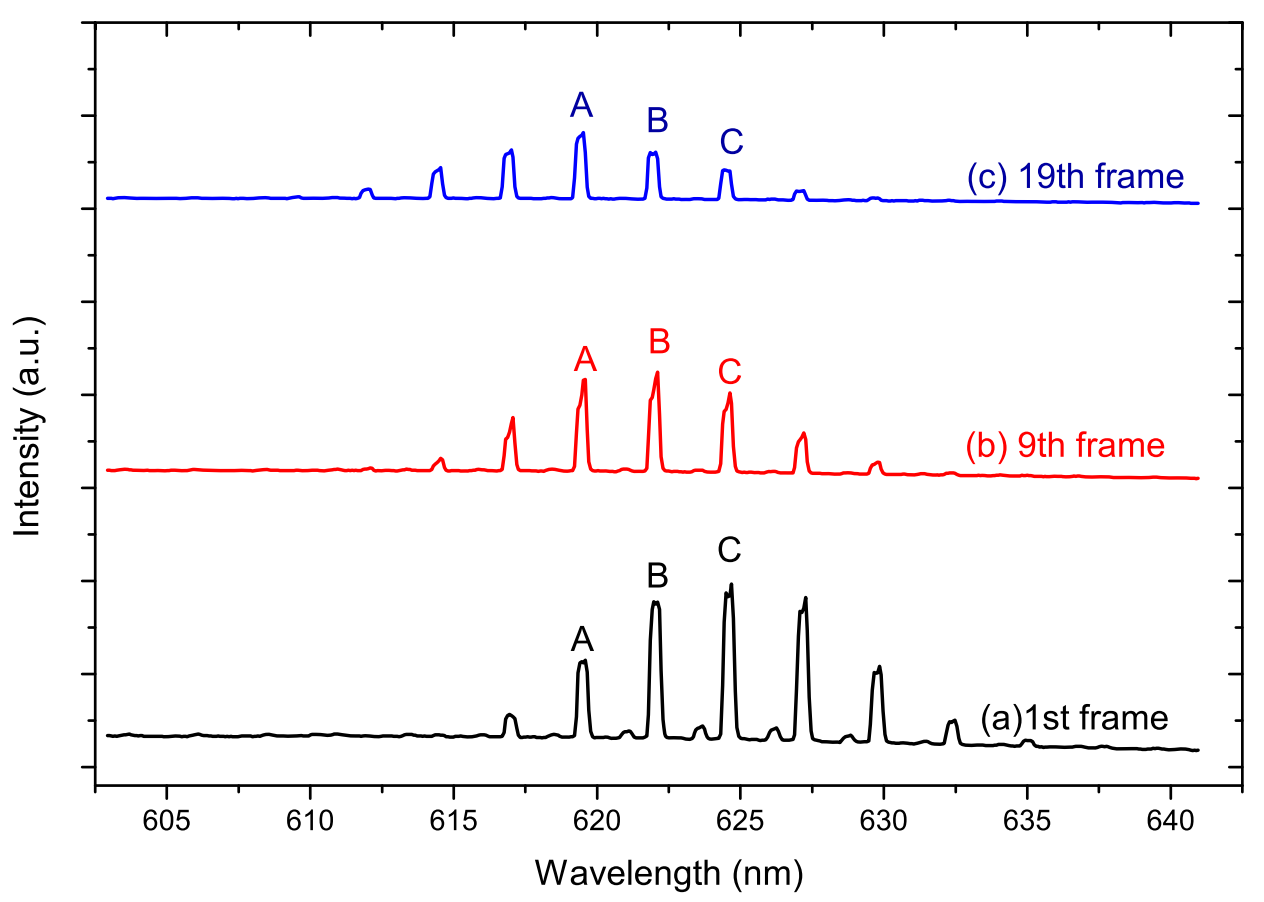

Figure 6: Effect of photobleaching on the droplet lasing spectra. 1st, 9th, and 19th frames of spectra acquired from $\mathrm{a} \sim 34 \mu \mathrm{m}$ diameter droplet are shown. Average pump power was $1.8 \mathrm{~mW}$ and exposure time for each of the frames was $10 \mathrm{~ms}$. 